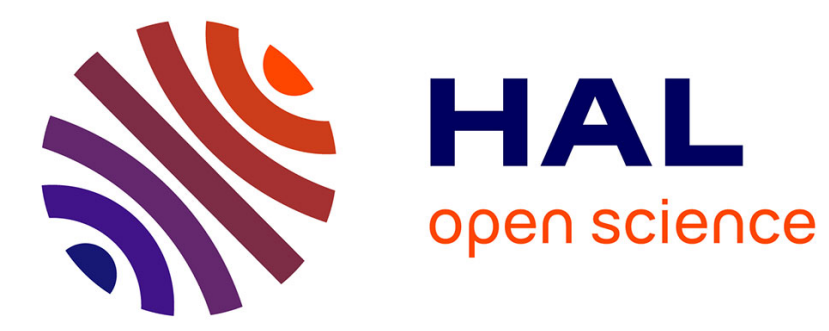

\title{
Topology Control in VANET and Capacity Estimation
} Anh Tuan Giang, Anthony Busson, Alain Lambert, Dominique Gruyer

\section{To cite this version:}

Anh Tuan Giang, Anthony Busson, Alain Lambert, Dominique Gruyer. Topology Control in VANET and Capacity Estimation. 2013 IEEE Vehicular Networking Conference (VNC), Dec 2013, BOSTON, United States. 10.1109/vnc.2013.6737600 . hal-00922261

\section{HAL Id: hal-00922261 \\ https://hal.inria.fr/hal-00922261}

Submitted on 30 Jan 2014

HAL is a multi-disciplinary open access archive for the deposit and dissemination of scientific research documents, whether they are published or not. The documents may come from teaching and research institutions in France or abroad, or from public or private research centers.
L'archive ouverte pluridisciplinaire HAL, est destinée au dépôt et à la diffusion de documents scientifiques de niveau recherche, publiés ou non, émanant des établissements d'enseignement et de recherche français ou étrangers, des laboratoires publics ou privés. 


\section{Topology Control in VANET and Capacity Estimation}

\author{
Anh Tuan GIANG \\ Laboratory of Signals and Systems \\ Université Paris Sud - Supélec - CNRS \\ Alain LAMBERT \\ IFSTTAR \\ IM, LIVIC \\ F-78000 Versailles, France
}

\author{
Anthony BUSSON \\ Laboratoire de l'Informatique du Parallélisme (LIP) \\ University Lyon 1 - ENS-LYON - INRIA \\ Dominique GRUYER \\ IFSTTAR \\ IM, LIVIC \\ F-78000 Versailles, France
}

\begin{abstract}
Some safety applications using VANET exchange a large amount of data, and consequently require an important network capacity. In this paper, we focus on extended perception map applications, that use information from local and distant sensors to offer driving assistance (autonomous driving, collision warning, etc). Extended perception requires a high bandwidth that might not be available in practice in classical IEEE 802.11p ad hoc networks. Therefore, we propose an adaptive power control algorithm optimized for this particular application. We show through an analytical model and a large set of simulations that the network capacity is then significantly increased.
\end{abstract}

\section{INTRODUCTION}

Perception map consists for a vehicle in collecting data through a set of embedded sensors measuring the surrounding environment. It gives both a local representation and modeling of the information resources used by the vehicle applications like ACC (Adaptive Cruise Control), Stop and Go, road departure avoidance, collision mitigation, etc. With the VANET, perception maps may be broadcasted to the adjacent vehicles allowing a node to extend its local vision. The so-called "extended perception" may improve the safety applications as it offers a better risk assessment, a better anticipation of dangerous situation, and may provide information for autonomous driving applications.

But, information from sensors needs to be exchanged at a high rate (up to 100 packets per second) to be pertinent. Therefore, extended perception may generate an important amount of data that must be efficiently carried by the network. The fundamental and natural question that arises is thus to know if the VANET can offer such a capacity. If not, we need to propose mechanisms offering enough bandwidth to support these essential applications.

In a near future, VANET should be based on the recent IEEE $802.11 \mathrm{p}$ [2] standard for its communication. The capacity of IEEE $802.11 \mathrm{p}$ based ad hoc networks is mainly limited by the spatial reuse, due to the CSMA/CA mechanism. Indeed, a node in a CSMA/CA network can access to the share wireless medium only if the medium is detected idle. To determine this idleness, the CSMA/CA uses a sub-mechanism named CCA (Clear Channel Assessment). It may report a busy medium upon detecting any energy above an Energy Detection threshold (CCA mode 1). In this case, the channel occupancy is related to the total interference level. Or, it may report a busy medium if it detects a signal compliant with its own standard (CCA mode 2), i.e. same physical layer (PHY) characteristics, such as modulation or spreading. Also, the wireless interface may use a logical combination (e.g. AND or OR) of the two previous mode (CCA mode 3). Obviously, these CCA mechanisms ensure that only nodes sufficiently far from the transmitting node can access the medium and limit interference between concurrent transmissions. But, it also limits the number of simultaneous transmitters, the number of frames that the network can carry per second, and consequently the VANET capacity.

A natural way to improve this capacity is to increase the spatial reuse by using an adaptive transmission power control algorithm. It is particularly pertinent in this context as the perception map application send information to the neighborhood in a sub-radio scope (around 100 meters) and not in the whole $802.11 \mathrm{p}$ radio range. A certain number of power control algorithm designed for 


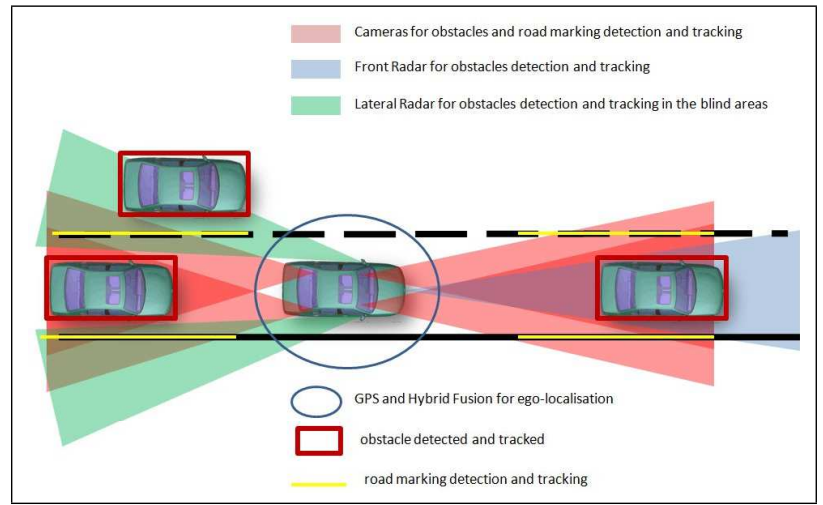

Fig. 1. Attributes of a perception local map.

VANET have been recently proposed [5], [16], [13], [4], [12], but they cannot be applied to the perception map application due to its specific constraints.

This paper aims to propose and evaluate an adaptive Transmission Power Control (TPC) algorithm designed for perception map applications. This application is presented in Section II The first contribution is thus a power control algorithm presented in Section IIII In Section IV] we propose an analytical model allowing us to evaluate the performance of our algorithm in term of capacity. Simulations and analytical results are compared in Section V, and we conclude in Section VI

\section{iI. Perception Map Application}

Recently, it is become very important to extend the perception range in order to anticipate the hazardous situation (risk assessment) and to provide information for autonomous driving applications (copilot application with path planning and navigation functionalities) [14]. The extended map modeling is both a spatial and temporal representation of a specific extended situation (limited in the local map by sensor ranges) allowed by communication means. An illustration of embedded sensors that can be equipped on a vehicle for building the perception map is depicted in Figure 1. By using communications within a range of less than 50-100 meters, we can send information to the other vehicles moving both in front and in the rear of our position. This local information can also be used in order to inform vehicles far away from the ego-vehicle (in rear position) to have enough information to assess a risk indication. Such an application has been already tested in [7], [6] and prove its efficiency to reduce the global risk point of view. In these papers, the authors compare the performance of a cooperative risk assessment using an extended map against a non-cooperative approach based on local-perception only. The results of this study

\begin{tabular}{|c|c|c|c|}
\hline Neighbor ID & Up-link & Down-link & local time out \\
\hline \hline 192.168 .0 .1 & $-75 \mathrm{dBm}$ & $-54 \mathrm{dBm}$ & timeout $_{1}$ \\
\hline 192.168 .0 .3 & $-60 \mathrm{dBm}$ & $-59 \mathrm{dBm}$ & timeout $_{2}$ \\
\hline$\cdots$ & $\cdots$ & $\cdots$ & $\cdots$ \\
TABLE I & $\cdots$ \\
EXAMPLE OF A LocalNeighborsList.
\end{tabular}

show a systematic improvement of forward warning time for most vehicles in a platoon scenario when using the extended-map-based risk assessment.

But, data quality heavily depends on both the quality of the local algorithms used to perceive the environment, and the communication capability to send an amount of data in a short time and in a dense traffic configuration. The more delay the communications have, the more uncertainties on the data (especially the position, speed, and heading) will be degraded and unusable. With the required frequency of exchanged between vehicles (up to $100 \mathrm{~Hz}$ ), and the expected radio range of the IEEE $802.11 \mathrm{p}$ technology (up to $1 \mathrm{~km}$ ), such application may not be supported due to the lack of network capacity [8]. The work presented in this paper is dedicated to this problem and try to give a first answer in order to improve the network capacity for a better extended perception.

\section{AdAPTIVE TPC Algorithm}

\section{A. Motivation}

Our power control algorithm is dedicated to the extended map application, i.e. transmission power changes apply only to this application packets. As described earlier, the perception map application have the following properties: each vehicle/node broadcasts information at a high frequency, information contained in these packets are pertinent in the vicinity of the nodes (50-100 meters), and the application does not require a fully reliable delivery of the broadcasted packets so it tolerates a few losses. Therefore, the proposed power control algorithm aims to ensure a good reception rate of broadcast packets for receivers lying less than a certain distance (denoted $d_{r e f}$ in the following), and with the smallest possible transmission power. We do not assume any particular radio environment, path-loss, etc. The algorithm is thus adaptive, i.e. transmission power is tuned only with regard to measures made locally on each node. Basically, the algorithm has three tasks: update a list of nodes at distance less than $d_{r e f}$, spy the reception qualities for these nodes, and increase/decrease the transmission power according to these information.

\section{B. Algorithm details}

The algorithm and the application assume that all involved nodes are equipped with GPS receiver or any 


\begin{tabular}{|c|c|}
\hline Parameters & Values \\
\hline \hline Application packet frequency & Varying \\
\hline HELLO_INTERVAL & 1 second \\
\hline LOCAL_TIMEOUT & $3 \times$ Packet frequency $(0.3 \mathrm{sec})$ \\
\hline GLOBAL_TIMEOUT & $3 \times$ HELLO_INTERVAL $(3.0 \mathrm{sec})$ \\
\hline$P_{\max }$ & 33 \\
\hline$\theta$ & $-90 \mathrm{dBm}$ \\
\hline$d_{\text {ref }}$ & 50 meters \\
\hline$\Delta$ & $1 \mathrm{dBm}$ \\
\hline \multicolumn{2}{|}{} \\
\hline
\end{tabular}

DEFAULT VALUES OF THE POWER CONTROL ALGORITHM.

devices allowing a node to know its location. Our algorithm manages two lists of neighbors.

The first list relies on a link sensing mechanism using HELLO packets. These HELLOs are sent periodically, at a low frequency denoted HELLO_INTERVAL (about 1 or 2 seconds), at the maximum transmission power, and include the sender location. It allows each node to keep a global neighbors list, with ID and locations of the neighbors. An entry/neighbor is removed from this list if no HELLO is received for a GLOBAL_TIMEOUT period. This algorithm being very classical, we do not present the details.

The second list contains only nodes at a distance less than $d_{\text {ref }}$ (pertinent distance from the application point of view). To manage this list, we use the packets of the perception map application that periodically broadcasted packets at a high frequency. The power control algorithm applies to these packets. The corresponding list of nodes is denoted LocalNeighborsList. It contains the neighbor IDs, up-link and down-link quality, and a local time out as shown in Table [I The initial local timeout is set according to the constant LOCAL_TIMEOUT. This timeout aims to update/remove an entry of the local neighbor list when there are several consecutive missed packets from this neighbor. The up-link and down-link qualities may be the received signal strength, SNR, SINR or any quantity reflecting the link quality. In our simulations, we considered the received signal strength since it is available, but for a real implementation the RSSI (Radio Signal Strength Indicator) could be considered instead. The down-link quality is updated at the reception of a probe/application packet. When sending a probe packet (an application packet using this algorithm is called a probe packet), the sender piggybacks its own location and its LocalNeighborList. These information allows the receiver to update the location/distance and the up-link quality for this neighbor.

The algorithm includes 3 sub-procedures. The three procedures are detailed in Table 1, 2, and 3. Each node
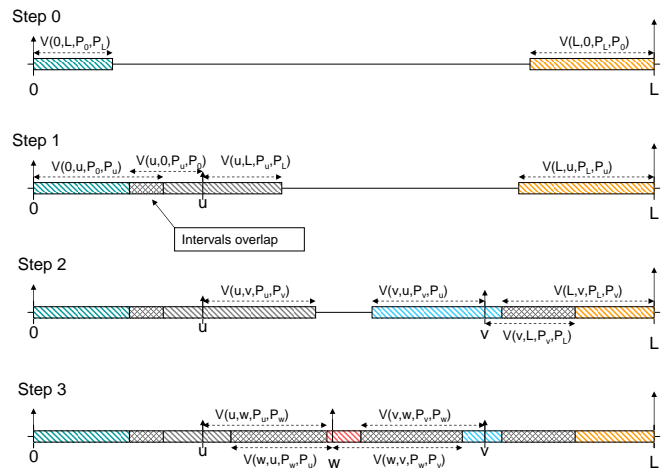

Fig. 2. Random packing model example.

has a global variable TxPower that sets the transmission power of the probe packets. When a node $\mathrm{R}$ receives a probe packet from a node $\mathrm{E}$, it calls the Reception() procedure. $\mathrm{R}$ updates the LocalNeighborsList, and increases the transmission power if $\mathrm{R}$ is not in LocalNeighborsList of $\mathrm{E}$ who sent this probe (meaning that the transmission power of $\mathrm{R}$ is not sufficiently high to reach its neighbor $\mathrm{E}$ ). When a node wants to transmit its probe, it calls the Transmission() procedure. It checks if the LocalNeighborsList contains all nodes at distance less than $d_{r e f}$. If not, it increases its transmission power. Also, it checks if all neighbors at distance less than $d_{r e f}$ received its probes with a minimum quality (denoted $\theta$ in the algorithm). If yes, it decreases the transmission power. The procedure LocalTimeoutExpiration(), called at the local time out expiration, aims to update the LocalNeighborList when a node is at distance greater than $d_{\text {ref }}$. The defaults values of the different parameters involved in our algorithm are given in Table II

\section{RANDOM PACKING MODEL}

\section{A. The model}

We propose in this Section a random packing model to evaluate the network capacity when using a power control algorithm. Packing model consists in placing points in a given space under some constraints until the space is completely filled. The considered space is $\mathbb{R}^{+}$, representing a road or a highway. In our context, points will represent the transmitter locations. We deduce the mean number of simultaneous transmitters, hence the mean number of transmitted frames and the capacity.

Most of the packing problem [9], [15] associate to each point an interval of fixed or random size. The next points are kept if and only if it does not lie in one of 

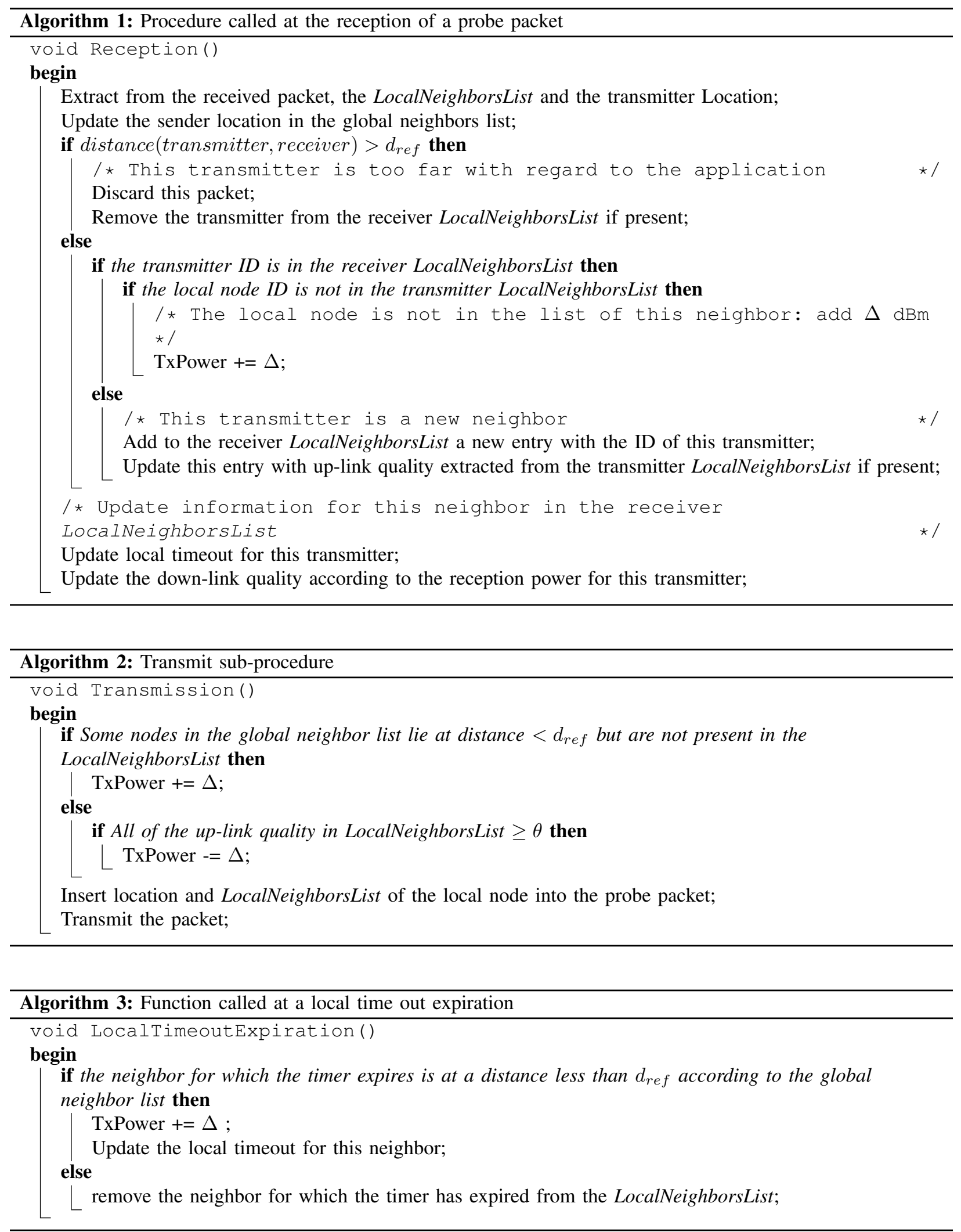
these interval, and so on. These processes can model the CCA mode 2, where the medium is set as busy when a signal compliant with the IEEE 802.11 standard is detected. Indeed, if we assume that a transmission/signal is detected at a certain distance, the interval of the car packing model represents the region around the transmitter where its signal is detected. But with the CCA mode 1 , a medium is sensed as busy or idle according to the interference signal strength. It does not correspond to the signal from the closest transmitter, but as the sum of the signals of the current transmissions. Extensions of the classical packing model have been proposed to model this mode [8], but they assume a constant transmission power. We propose here, a packing process modeling mode 1 with heterogeneous transmission power.

With CCA mode 1, the medium is busy if this interference level is above a pre-defined threshold denoted $\gamma$. We assume that radio environment can be modeled by a pathloss function, denoted $l($.$) . It gives the received signal$ strength according to the distance from the transmitter when the transmitting power is $1(\mathrm{~mW}$ or $\mathrm{W}) . l($.$) , de-$ fined in $\mathbb{R}^{+}$, is assumed positive, continuous, decreasing with $\lim _{x \rightarrow+\infty} l(x)=0$.

Due to the fact that radio signals attenuate quickly with the distance, interference is mainly composed of the closest interferers. Therefore, our model takes into account the interference that is generated by the two closest transmitting nodes, from the left and from the right. Interference at location $x$ is thus formally described as:

$$
I(x)=P_{l e} l(x-l e)+P_{r i} l(x+r i)
$$

where $l e$ and $r i$ are the locations of the two closest interferers on left and right hand sides of $x . P_{l e}$ (resp. $P_{r i}$ ) is the transmitting power from node at le (resp. ri). $P_{y}$ is thus a random variable describing the transmission power for a node at location $y$. Transmitting powers are assumed i.i.d., and greater than $\gamma$ almost surely (the transmitting power is greater than the CCA detection threshold).

Our model is built as follows. We consider an interval $[0, L]$ with $L \in \mathbb{R}^{+}$. We assume that there are two points/nodes at 0 and $L$. For $L$ sufficiently great, there are two sub-intervals denoted $\left[0, v\left(0, L, P_{0}, P_{L}\right)\right]$ and $\left[0, v\left(L, 0, P_{L}, P_{0}\right)\right]$ (represented in Figure 2p where the interference level is greater than $\gamma$. These busy intervals cannot host new transmitters as they will detect a busy medium. Their formal definitions are described below. If these two subinterval does not overlap, there is an idle interval where a new transmitter/point can be added. It is uniformly distributed in this interval. It corresponds to the step 1 in Figure 2. This new point, located at $u$ in our example, generates two busy intervals of lengths $v\left(u, 0, P_{u}, P_{0}\right)$ and $v\left(u, L, P_{u}, P_{L}\right)$ respectively. Also, the lengths of the busy intervals at 0 and $L$ increase since the interferer at $u$ is closer. The intervals become $v\left(0, u, P_{0}, P_{u}\right)$ and $v\left(L, u, P_{L}, P_{u}\right)$. Then, a new point is added in the idle interval (at $v$ in step 2), and so on. We repeat this process until there is no idle interval in $[0, L]$.

The busy intervals $v(., ., .,$.$) are defined as follows.$ $v\left(\right.$ loc $_{1}$, loc $_{2}$, power $_{1}$, power $\left._{2}\right)$ represents the length of the busy interval around location $l o c_{1}$ when interferers are located at distance $l o c_{1}$ and $l o c_{2}$ with transmitting powers power $_{1}$ and power $_{2}$. This interval is located on the right hand side of $l o c_{1}$ when $l o c_{2}>l o c_{1}$ and on the left hand side otherwise. It is formally defined as the solution of $I\left(l_{o c} c_{1}+\right.$ $v\left(\right.$ loc $_{1}$, loc $_{2}$, power $_{1}$, power $\left.\left._{2}\right)\right)=\gamma$ when $l o c_{2}>l o c_{1}$ and $I\left(\operatorname{loc}_{1}-v\left(\operatorname{loc}_{1}, \operatorname{loc}_{2}\right.\right.$, power $_{1}$, power $\left.\left._{2}\right)\right)=\gamma$ otherwise.

Proposition 1: Let $m(L)$ be the mean number of points in the interval $(0, L)$ (we do not count the two points at 0 and $L$ ) for the process defined above, then:

$$
\lim _{L \rightarrow \infty} \frac{m(L)}{L}=\beta
$$

where $\beta$ is a positive constant.

By lack of place, we give only the sketch of the proof. We can observe that $m(L)$ is a super-additive function, i.e. $m(L) \geq m(s)+m(L-s)$. According to the Fekete's lemma, $\frac{m(L)}{L}$ converges to a finite constant or diverges to infinity. To conclude it suffices to show that there exists a constant $A$ such that $m(L)<A \cdot L$ for all $L$. It is straightforward since there is a minimal distance between the points. $\frac{m(L)}{L}$ is thus bound, and converges to a finite limit.

\section{B. Capacity estimation}

The positive constant $\beta$ can be used to estimate the mean number of simultaneous transmitters over a road with length $L$ at a given time. We denote $T$ the mean time to transmit a $802.11 \mathrm{p}$ frame. It takes into account the different times used in the $802.11 \mathrm{p}$ protocol (DIFS, SIFS, etc.). We do not consider acknowledgment as our application generates only broadcast traffic. The average number of frames that a network with length $L$ can transmit per second can be expressed as:

$$
\operatorname{Capacity}(L)=\frac{\beta L}{T}
$$

According to equation (3), estimation of the capacity boils down to the computation of the limit $\beta$. We propose an estimation that allows us to compute this constant 


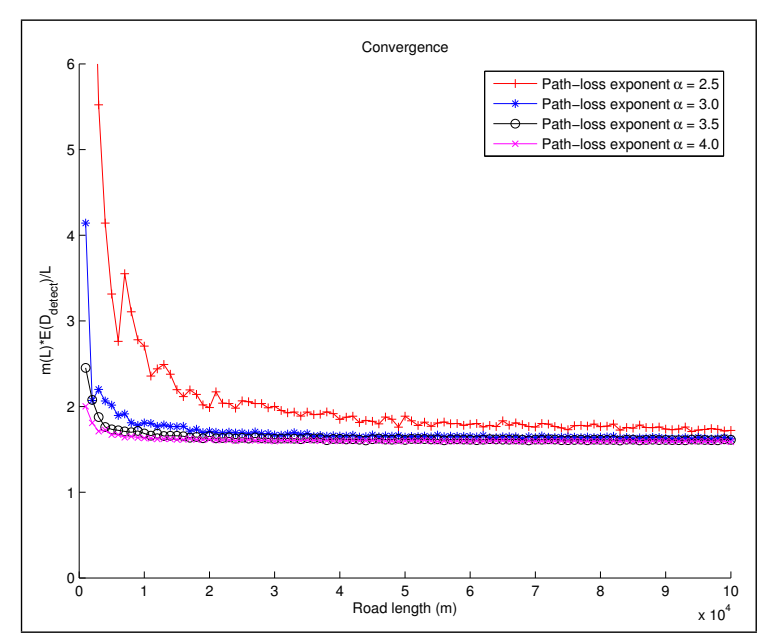

Fig. 3. Convergence of $\frac{m(L) \mathbb{E}\left[D_{\text {detect }}\right]}{L}$ for different path-loss function parameters.

from the path-loss function and the distribution of the transmission power. Since the adaptive TPC algorithm leads to the use of different transmission powers, we represent it as a random variable $P_{t x}$. We collected more than 120,000 samples of transmission powers from simulations (described in a next section). The best fit among the classical distributions of the empirical distribution of $P_{t x}-P_{\max }$, where $P_{\max }$ is the maximum transmission power, was the exponential law. Therefore, we express the transmission power distribution as a shifted exponential random variable truncated on the interval $\left[0, P_{\max }\right]$. Its p.d.f. is given by:

$$
f_{P_{t x}}(x)=\frac{\lambda}{1-e x p^{-\lambda P_{\max }}} \exp ^{-\lambda\left(P_{\max }-x\right)}
$$

$\lambda$ has been inferred from the samples. In order to estimate $\beta$, we consider the mean detection distance denoted $D_{\text {detect }}$. It corresponds to the distance at which a node detects a transmission when there is no other sources of interference. $D_{\text {detect }}$ is the solution of $P_{t x} l\left(D_{\text {detect }}\right)=$ $\gamma$. We get:

$$
D_{\text {detect }}=l^{-1}\left(\frac{\gamma}{P_{t x}}\right)
$$

In Figure 3, we plotted the quantity $\frac{m(L) 2 \mathbb{E}\left[D_{\text {detect }}\right]}{L}$ when $L$ increases $\left(\mathbb{E}\left[D_{\text {detect }}\right]\right.$ is the expectation of $\left.D_{\text {detect }}\right)$. Each point is the average of 100 samples and is shown with a confidence interval at $95 \%$. The considered path-loss function is the classical log distance path-loss [11]: $l(d)=\min \left(c, c / d^{\alpha}\right)$. The values of the parameters are given in Table III. We observe that all curves converge to the same constant, approximately equal to 1.70 . This result is not surprising as it holds for other packing problems in one or two-dimensional spaces (see [9] or [3] for instance). This convergence to a universal constant allows us to estimate the limit $\beta$ of Proposition 1 as follows:

$$
\lim _{L \rightarrow+\infty} \frac{m(L)}{L}=\frac{1.70}{\mathbb{E}\left[D_{\text {detect }}\right]}
$$

Therefore, the final capacity can be expressed as:

$$
\operatorname{Capacity}(L)=\frac{1.70 L}{\mathbb{E}\left[D_{\text {detect }}\right] T}
$$

\section{NS-3 Simulation RESUlTS}

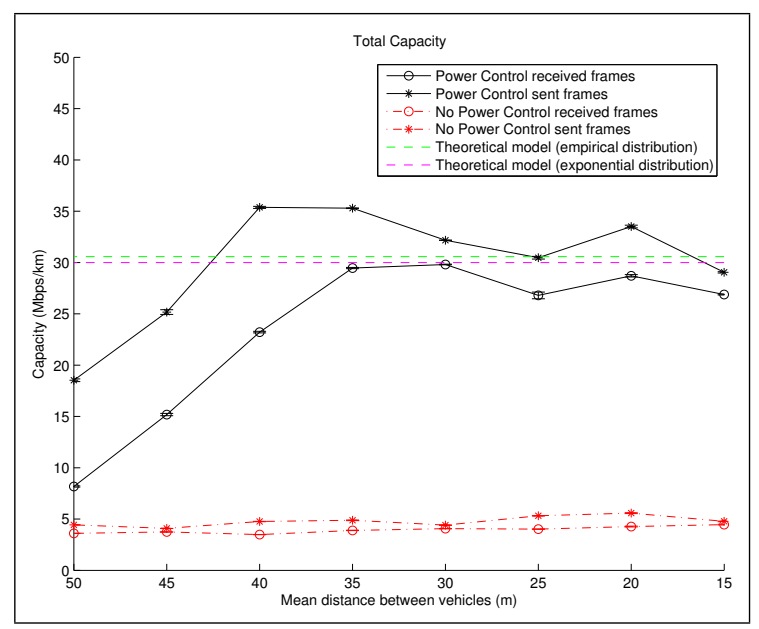

(a) Constant Total Capacity

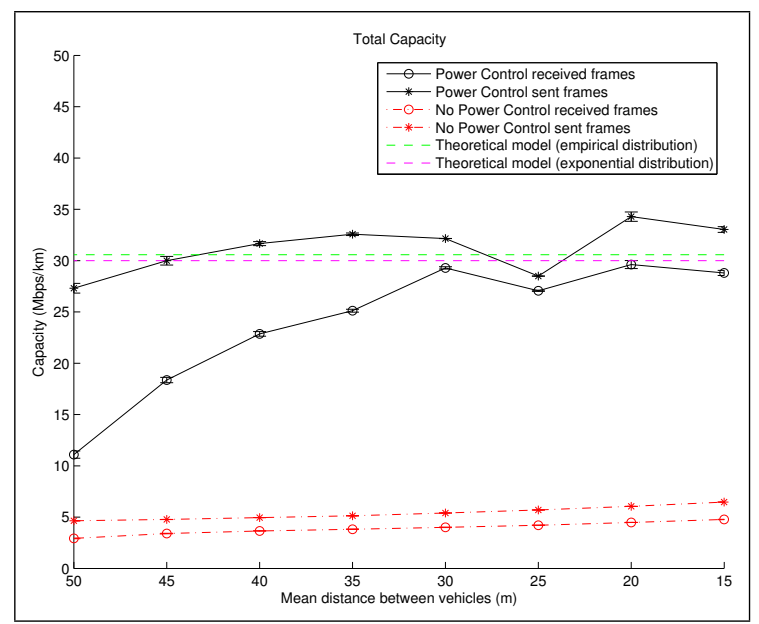

(b) Mobile Total Capacity

Fig. 4. Total capacity for constant and mobile case in pure broadcast scenarios.

In order to validate our theoretical model and study the performance of our algorithm, we implemented our algorithm in NS-3 [1]. In these simulations, nodes were 


\begin{tabular}{|l|l||l|l|}
\hline Theoretical and NS-3 Parameters & Numerical Values & Theoretical and NS-3 Parameters & Numerical Values \\
\hline \hline IEEE $802.11 \mathrm{std}$ & $802.11 \mathrm{p}-\mathrm{CCH}$ channel & Path-loss function & $l(d)=P_{t} \cdot \min \left(1, \frac{10^{-4.5677}}{d^{3}}\right)$ \\
CCA mode & CCA mode 1 & CCA ED Threshold $(\gamma)$ & $-99 \mathrm{dBm}$ \\
Road length (L) & $15 \mathrm{~km}$ & Number of samples per point & 100 \\
Length of the packet & 1024 bytes & Duration of the simulation & $3 \mathrm{sec}$ \\
DIFS (DCF Interframe Space) & $34 \mu \mathrm{s}$ & SIFS (Short Interframe Space) & $16 \mu \mathrm{s}$ \\
\hline
\end{tabular}

SIMULATION PARAMETERS.

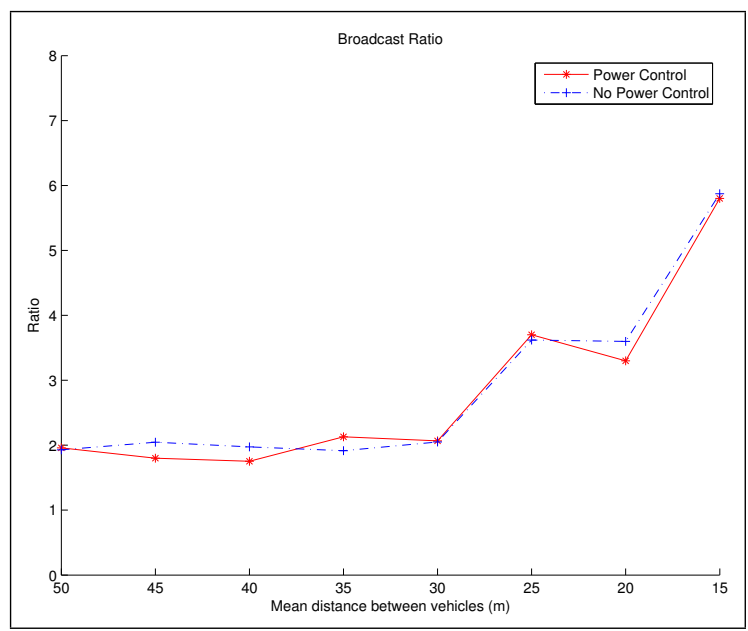

(a) Constant broadcast ratio

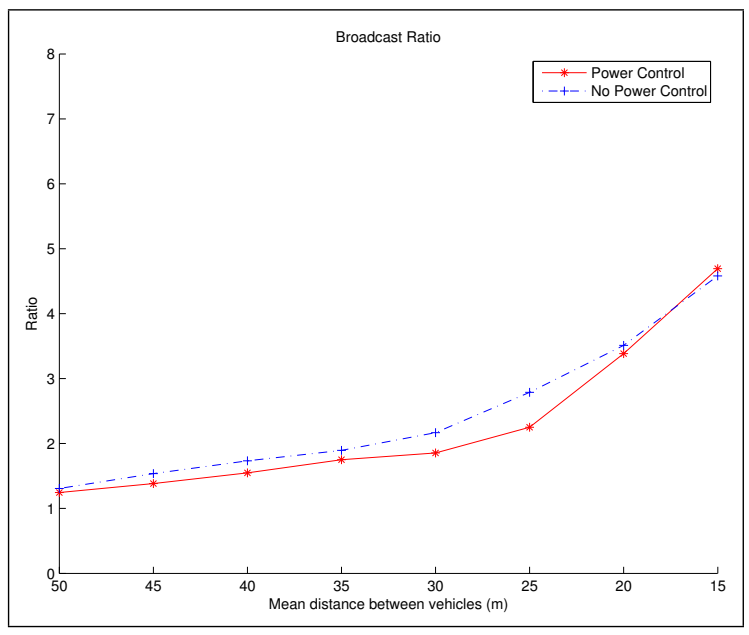

(b) Mobile broadcast ratio

Fig. 5. Broadcast ratio for constant and mobile case in pure broadcast scenarios. equipped with IEEE $802.11 \mathrm{p}$ interfaces. The parameters are given in Table III Vehicles were located along a line modeling a highway of $15 \mathrm{~km}$. Vehicles periodically broadcast probe packets using the TPC algorithm as defined in Section [III. These simulations aim to estimate the maximum rate reachable by our TPC algorithm. In order to estimate this maximum capacity we had to consider two different application rates. Indeed, as we increase the number of vehicles, a constant rate led to significant contention and a poor throughput when the traffic density became high. To keep a reasonable delivery rate, the number of packets per second generated by the perception map application was 125 packets/sec for inter-vehicle distances from 50 to 25 meters. For lower inter-distances, the application rate was 58 packets/sec.

Each point in the different figures are computed as the mean of 100 simulations and are presented with a confidence interval at $95 \%$. We considered 2 mobility scenarios: "constant mobility" where vehicles have a 104 $\mathrm{km} / \mathrm{h}$ constant velocity, and "Gaussian mobility" where the vehicle speeds follow a Gaussian distribution with mean $104 \mathrm{~km} / \mathrm{h}$ and variance 43 . These values have been set according to vehicle speeds collected on Canadian highways [10].

In order to evaluate the benefit of our TPC algorithm, we perform the same simulations with and without power control. The first quantity we considered is the broadcast ratio defined as the ratio of received frames over the number of sent frames. The number of received frames is the sum of the successful receptions for vehicles at distance less than $d_{r e f}$ from the transmitter. This quantity is thus greater than one, and increases with the vehicles density. In Figure 5, we plotted the broadcast ratio for the two mobility models. It shows that the delivery rate is almost the same with and without the power control algorithm. It means that our mechanism decreases the transmission power while keeping the targeted neighbors in its radio range. We have only a few losses with the Gaussian mobility model when the traffic becomes dense. These losses are mainly due to new neighbors entering within the pertinent area (distance 
$\left.<d_{\text {ref }}\right)$, that are not taken into account by our algorithm instantaneously.

The second quantity that we estimated is the spatial capacity. It is computed as the mean number of sent/received bits per second and per kilometer. When we consider the sent bits, we just count what is transmitted by the nodes. For the reception, we take into account bits of a broadcasted frame only once (even if there are several receptions), and only if it has been properly received by at least one node. The capacity improvement is shown in Figure 4 We observe a huge improvement of capacity (almost 10 times in some points). The fluctuation that appears when the inter-distance is equal to 25 meters is due to the change of our application rate explained earlier. The two horizontal lines correspond to the theoretical evaluation. We considered an exponential distribution of the transmission powers, and the empirical distribution obtained from the simulation samples. The bound from the exponential distribution is very close to the empirical one, and has the benefit to be easily and analytically computable. The two bounds are very accurate. For some points, the number of sent bits is greater than the theoretical bounds, but it is due to collisions, i.e. when a transmission does not respect the CCA rules (mainly due to the draw of the same back-off by two nodes). But, when we are looking at the number of received bits, our bounds are not reached and clearly offer very good estimates.

\section{CONCLUSION}

In this paper, we propose an adaptive TPC algorithm dedicated to extended perception map building. It is based on signal strength measurements of the packets generated by the application. It is worth noting that without power control, the perception map application is likely unusable by lack of capacity. We have shown through simulations and a theoretical model that this algorithm may improve the network capacity up to 10 times. It offers an extended bandwidth while keeping a good transmission reliability.

\section{REFERENCES}

[1] Network simulator 3 - ns3. http://www.nsnam.org

[2] Lee Armstrong and Wayne Fisher. Status of project ieee 802.11 task group p. wireless access in vehicular environments (wave). http://grouper.ieee.org/groups/802/11/Reports/tgp_update.htm

[3] A. Busson and G. Chelius. Point processes for interference modeling in csma/ca ad-hoc networks. In Sixth ACM International Symposium on Performance Evaluation of Wireless Ad Hoc, Sensor, and Ubiquitous Networks (PE-WASUN 2009), Tenerife, Spain, October 2009.

[4] Lin Cheng and R. Shakya. Vanet adaptive power control from realistic propagation and traffic modeling. In Radio and Wireless Symposium (RWS), 2010 IEEE, pages 665-668, 2010.
[5] Chunxiao Chigan and Jialiang Li. A delay-bounded dynamic interactive power control algorithm for vanets. In Communications, 2007. ICC '07. IEEE International Conference on, pages 5849-5855, 2007.

[6] S. Demmel, D. Gruyer, and A. Rakotonirainy. Simulation architecture for cooperative its applications and augmented perception. In OPTIMUM 2013.

[7] S. Demmel, G. Larue, and D. Gruyer. An ieee $802.11 \mathrm{p}$ empirical performance model for cooperative systems applications. In 16th International IEEE Annual Conference on Intelligent Transportation Systems (IEEE ITSC 2013), 2013.

[8] A.T. Giang, A. Busson, D. Gruyer, and A. Lambert. A packing model to estimate vanet capacity. In Wireless Communications and Mobile Computing Conference (IWCMC), 2012 8th International, pages 1119-1124, 2012.

[9] P. Hall. Introduction To the Theory of Coverage Processes. Wiley, 1988.

[10] J Macangus, C Milligan, J Montufar, and L BELLUZ. Speed characteristics on manitobas national highway system roads using weigh-in-motion data. In 2012 Conference and exhibition of the transportation association of Canada-transportation: innovations and opportunities, 2012.

[11] Theodore S Rappaport et al. Wireless communications: principles and practice, volume 2. Prentice Hall PTR New Jersey, 1996.

[12] Jun Tian and Chao Lv. Connectivity based transmit power control in vanet. In Wireless Communications and Mobile Computing Conference (IWCMC), 2012 8th International, pages 505-509, 2012.

[13] M. Torrent-Moreno, J. Mittag, P. Santi, and H. Hartenstein. Vehicle-to-vehicle communication: Fair transmit power control for safety-critical information. Vehicular Technology, IEEE Transactions on, 58(7):3684 -3703, sept. 2009.

[14] B. Vanholme, D. Gruyer, B. Lusetti, S. Glaser, and S. Mammar. A legal safety concept for highly automated driving on highways. In IEEE Transaction on Intelligent Transportation Systems, march 2013.

[15] Howard J Weiner. Sequential random packing in the plane. Journal of Applied Probability, pages 803-814, 1978.

[16] Lin Yang, Jinhua Guo, and Ying Wu. Channel adaptive one hop broadcasting for vanets. In Intelligent Transportation Systems, 2008. ITSC 2008. 11th International IEEE Conference on, pages 369-374, 2008. 